\title{
Dielectric properties of polyamide 12-chromium (III) oxide nanocomposites
}

\author{
E. S. Shapoval ${ }^{1}$, V. V. Zuev ${ }^{1,2}$ \\ ${ }^{1}$ ITMO University, Kronverkskiy pr., 49, 197101 St. Petersburg, Russia \\ ${ }^{2}$ Institute of Macromolecular Compounds of the Russian Academy of Sciences, \\ Bolshoi pr., 31, 199004 St. Petersburg, Russia \\ katenka-shapoval@yandex.ru; zuev@hq.macro.ru
}

\section{PACS 81.07.Lb}

DOI 10.17586/2220-8054-2016-7-3-472-478

\begin{abstract}
Broadband dielectric spectroscopy was employed to study polymer nanocomposites based on PA12 filled with different loading (0.1 - 10 wt.\%) of nanosized (average grain size of about $1-5 \mathrm{~nm}$ ) chromium (III) oxide. The experimental dielectric data were analyzed within the formalisms of complex permittivity and electric modulus. Three relaxation processes and Maxwell-Wagner-Sillars (MWS) interfacial polarizations were observed. It was found that all the relaxations were sensitive to filler contents. The presence of nanosized amphoteric chromium (III) oxide was shown to lead to the softening of the polyamide matrix that manifested in decrease of the activation energy of the $\alpha$ - and $\beta$-relaxation processes and glass transition temperatures. The softening of polymer matrix is the reason for the decrease in the mechanical properties of the polymer nanocomposites as compared to that of neat PA12.
\end{abstract}

Keywords: nanocomposites, polyamide 12, chromium (III) oxide, relaxation processes, activation energy dielectric spectroscopy.

Received: 8 February 2016

Revised: 19 March 2016

\section{Introduction}

Polymer magnetic materials can potentially find a great number of applications in different fields such as data storage, biomedicine, biosensors, drug delivery agent, magnetic resonance imaging devices, and a range of others [1-4]. Theoretical modeling predicts that the mechanical properties of polymer nanocomposites should be superior to those of conventional composites [5,6]. These improved properties are attained at lower filler content in comparison to conventionally filled polymers. However, the introduction of nanosized particles of magnetic oxides into different polymer matrices leads to a decrease in the mechanical properties. This was shown for the introduction of nanosized oxide $\mathrm{F}_{3} \mathrm{O}_{4}$ into polyurethane [7]. We obtained similar results for the preparation of polymer nanocomposites based on PA12 filled with different loading $(0.1-10 \mathrm{wt} . \%)$ of nanosized (average grain size of about $1-5 \mathrm{~nm}$ ) chromium (III) oxide [8]. It was suggested that the introduction of new fracture mechanisms, rigidity augmentation, and pronounced filler-filler interactions were responsible for the observed behavior instead of filler-polymer interactions [9-12]. The dynamics of polymer matrix changes due to changes in interactions with nanosized filler particles. The polymer dynamics and the glass transition in polymer nanocomposites are more complex than in neat polymer [13]. Here, we use broadband dielectric spectroscopy (BDS) to directly measure the influence of nanoparticles on polymer relaxations corresponding to different lengths and time scales. BDS is one of the most efficient tools for studying the molecular relaxations of polymers. It covers a broad frequency range, allowing measurement of different relaxation processes simultaneously, and even entire chain relaxation processes under favorable circumstances [14]. Relaxation processes in a polymer matrix are clearly connected with its mechanical properties. The $\beta$-relaxation is phenomenologically linked to the mechanical properties of polymeric materials $[15,16]$. The $\alpha$-relaxation is connected with the onset of large-scale motions of the chain segments in the vicinity of $T_{g}$ and determined viscoelastic behavior. The aim of the present work is to provide a description of the relaxation processes for $\mathrm{PA} 12 / \mathrm{Cr}_{2} \mathrm{O}_{3}$ nanocomposites over a range of temperatures and frequencies. As with any thermoplastic nanocomposites, a description of the relaxation processes parameters is of great interest, and is central for rational approach to thermomechanical processing.

\section{Experimental}

PA12/ $\mathrm{Cr}_{2} \mathrm{O}_{3}$ nanocomposites were synthesized according [8]. Dielectric measurements were performed using an Alpha Analyzer combined with a Quatro Temperature Control system unit that provides temperature stability of $0.1{ }^{\circ} \mathrm{C}$, both by Novocontrol. Complex dielectric permittivity $\varepsilon^{*}(f)=\varepsilon^{\prime}(f)-i \varepsilon^{\prime \prime}(f)$ was measured isothermally in steps of $5{ }^{\circ} \mathrm{C}$ from -150 to $+200{ }^{\circ} \mathrm{C}$ and over frequencies ranging from $10^{-2}$ to $10^{6} \mathrm{~Hz}$. The nanocomposite 
films were placed and melted in a parallel-plate copper capacitor with $20 \mathrm{~mm}$ diameter, and a pair of glass fiber with $80 \mu \mathrm{m}$ diameter was used as the spacers between electrodes.

\section{Results and discussion}

Plots of the three-dimensional real and imaginary parts, $\varepsilon^{\prime}$ and $\varepsilon^{\prime \prime}$, of the complex dielectric permittivity $\varepsilon^{*}$ versus frequency and temperature are presented in Fig. 1 for the sample with 5 wt. $\%$ of $\mathrm{Cr}_{2} \mathrm{O}_{3}$.

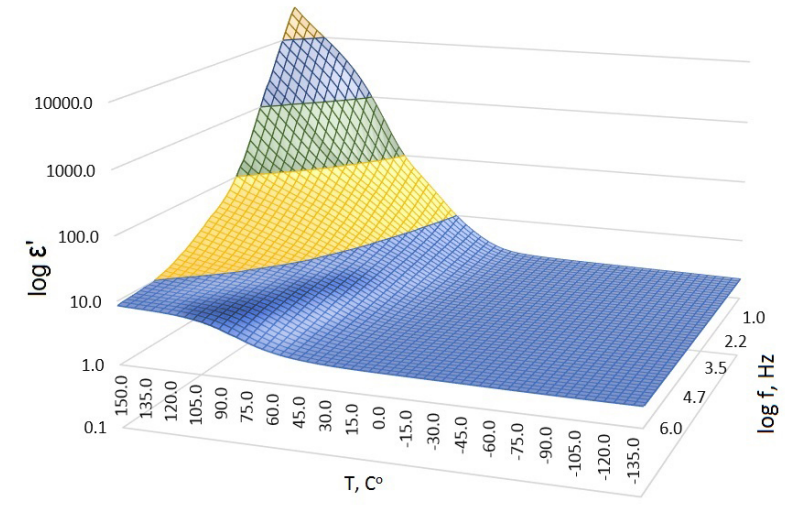

(a)

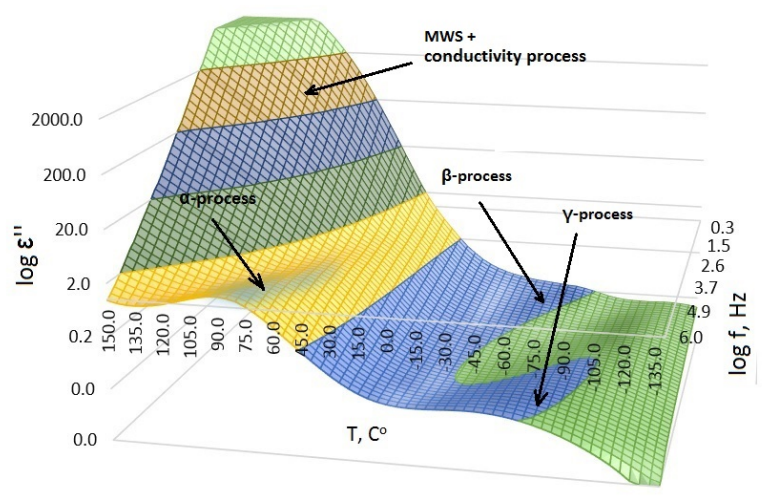

(b)

FIG. 1. 3D plot of $\varepsilon^{\prime}$ (a) and $\varepsilon^{\prime \prime}$ (b) versus frequency and temperature for nanocomposite with 5 wt. $\%$ of nanosized $\mathrm{Cr}_{2} \mathrm{O}_{3}$

The main relaxation processes identified are the same as those found for pure PA12 [17]: (1) the $\gamma$-relaxation appeared between $170 \mathrm{~K}$ and $250 \mathrm{~K}$; (2) the $\beta$ - relaxation appeared between $230 \mathrm{~K}$ and $275 \mathrm{~K}$; (3) the segmental $\alpha$-relaxation occurred between $270 \mathrm{~K}$ and $350 \mathrm{~K}$; (4) the MWS/conductivity process visible in the high temperature regime arising from the drift motion of the charges and charge carriers blocked at the interphase between amorphous and crystalline regions and conductivity effects. The four processes are typically for PAs [18] and were observed in all studied samples.

The isochronal graph at $1 \mathrm{kHz}$, Fig. 2, shows the temperature dependence of $\varepsilon^{\prime}$ and $\varepsilon^{\prime \prime}$ for neat PA12 and composites. The differences between samples are noted, especially at higher temperatures, where the MWS/conductivity process is located.

It should be noted that in the $\varepsilon^{\prime \prime}$ (f) plots (Fig. 2b) only an $\alpha$ loss peak was observed. MWS relaxation can be masked by large conduction effects at high temperatures. Moreover, the peak is superimposed by electrode

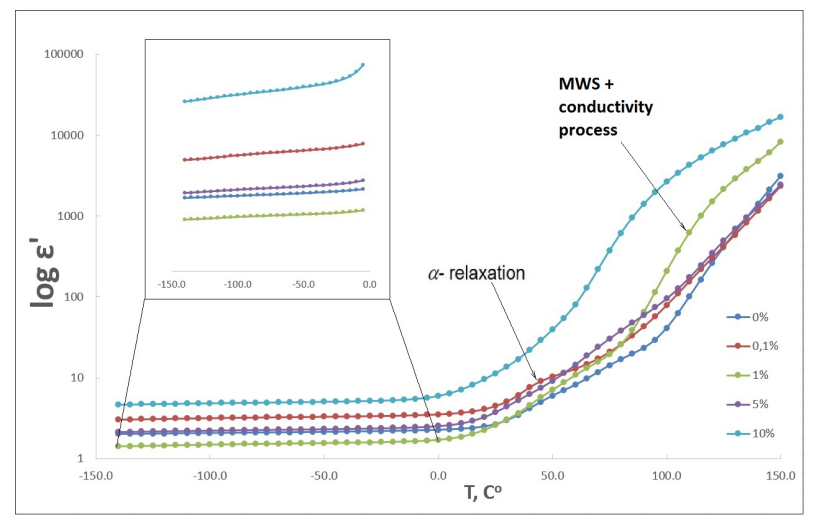

(a)

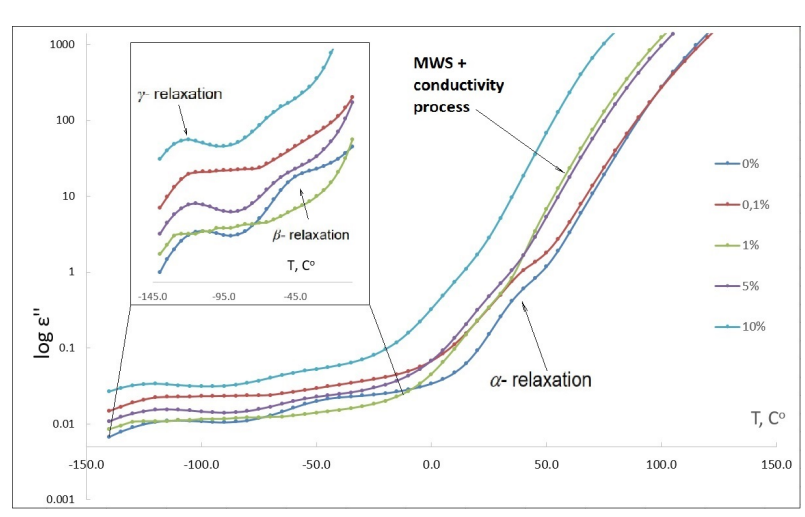

(b)

FIG. 2. Temperature dependence of $\varepsilon^{\prime}$ (a) and $\varepsilon^{\prime \prime}$ (b) for neat PA12 and all nanocomposites at a frequency of $1 \mathrm{kHz}$ 
polarization effects, and therefore not be clearly extracted in the permittivity spectra. Using the complex electric modulus formalism the MWS/conductivity process is visible as a peak in the imaginary part $M^{\prime \prime}$ of the complex dielectric modulus $M^{*} . M^{*}$ is related to $\varepsilon^{*}$ as follows [19]:

$$
\begin{gathered}
M^{*}=\frac{1}{\varepsilon^{*}}=M^{\prime}+i M^{\prime \prime}, \\
M^{\prime}=\frac{\varepsilon^{\prime}}{\varepsilon^{\prime 2}+\varepsilon^{\prime \prime 2}} \quad \text { and } \quad M^{\prime \prime}=\frac{\varepsilon^{\prime \prime}}{\varepsilon^{\prime 2}+\varepsilon^{\prime \prime 2}} .
\end{gathered}
$$

A plot of $M^{\prime \prime}$ versus frequency and temperature for the sample with 5 wt. $\%$ of $\mathrm{Cr}_{2} \mathrm{O}_{3}$ is shown in Fig. 3 .

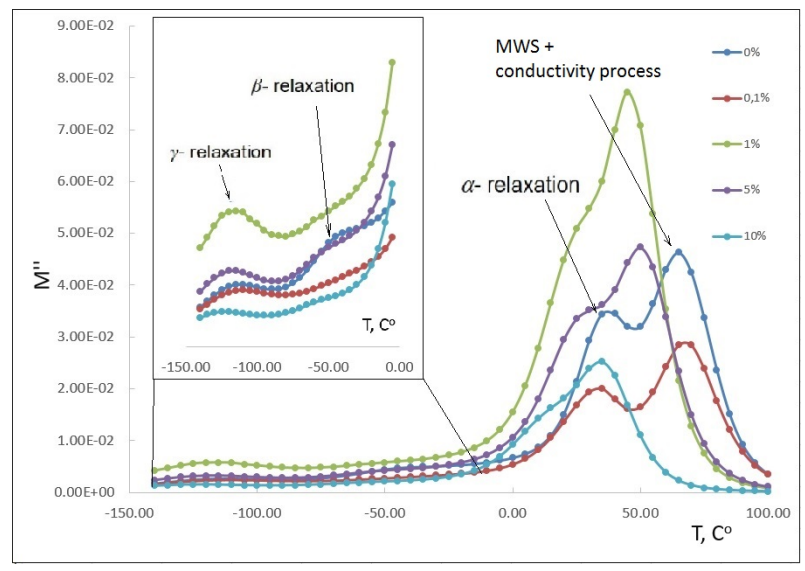

(a)

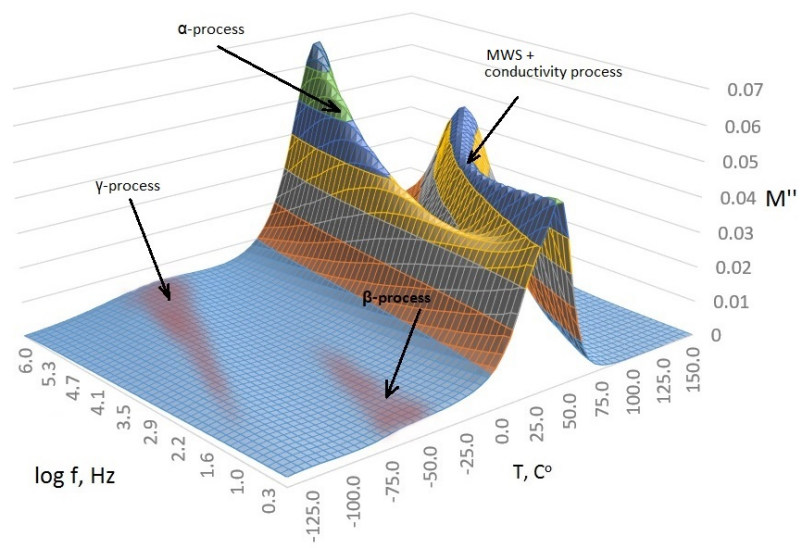

(b)

FIG. 3. (a) 3D plot of $M^{\prime \prime}$ versus frequency and temperature for nanocomposite with 5 wt.\% of nanosized $\mathrm{Cr}_{2} \mathrm{O}_{3}$ and (b) temperature dependence of $M^{\prime \prime}$ for neat PA12 and all nanocomposites at a frequency of $1 \mathrm{kHz}$

The MWS/conductivity process can now be identified as a peak in the higher frequency regime. In order to evaluate the individual relaxation processes quantitatively, a model function has been fitted to the dielectric data, with the Havriliak-Negami (H-N) phenomenological relation [20]:

$$
\varepsilon^{*}(\omega)=\varepsilon_{\infty}+\frac{\Delta_{\varepsilon}}{\left(1+\left(i \omega \tau_{N H}\right)^{1-\alpha}\right)^{\beta}},
$$

in its most general form. In this expression, $\varepsilon^{*}=\varepsilon^{\prime}-i \varepsilon^{\prime \prime}$, is the complex dielectric function, $\omega=2 \pi f, f$ is the field frequency, $\Delta_{\varepsilon}$ is the intensity of the dielectric process, $\tau_{N H}=1 / 2 \pi f_{N H}$ and $f_{N H}$ is the position of the relaxation process on the frequency scale, $\varepsilon_{\infty}$ is $\varepsilon^{\prime}(f)$ for $f \gg f_{N H}, \alpha$ and $\beta$ are shape parameters representing the symmetrical and asymmetrical broadening of the relaxation with respect to the Debye peak. The main characteristic of each relaxation process is the most probable relaxation time, $\tau_{\max }$, determined according to [21] as

$$
\tau_{\max }=\tau_{H N}\left(\frac{\sin \left(\frac{\pi \alpha \beta}{2(\beta+1)}\right)}{\sin \left(\frac{\pi \alpha}{2(\beta+1)}\right)}\right)^{1 / \alpha} .
$$

Figure 4 shows examples of such fits to the composites' relaxation processes at given temperatures for each process in the measured frequency window.

The $\alpha$-relaxation is associated with the onset of large-scale motions of the chain segments in the vicinity of $T_{g}$. At higher temperatures, near the $\alpha$-relaxation process, heating is accompanied by the creation of carriers due to the ionization of impurities and breaking of chemical bonds (the N-H bonds, etc). Hence, it is necessary to add an additional term related to the conductivity in Eq. (1):

$$
\varepsilon^{*}(\omega)=\varepsilon_{\infty}+\frac{\Delta_{\varepsilon}}{\left(1+\left(i \omega \tau_{N H}\right)^{1-\alpha}\right)^{\beta}}-i\left(\frac{\sigma_{0}}{\omega \varepsilon_{0}}\right) .
$$




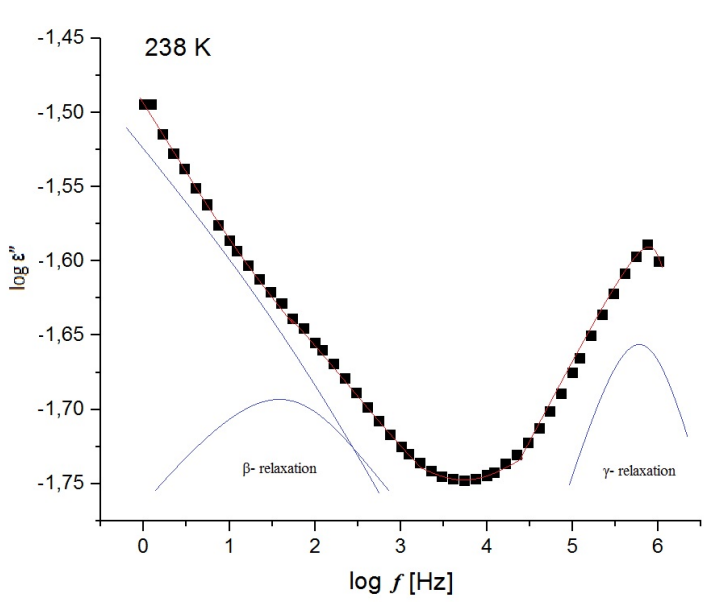

(a)

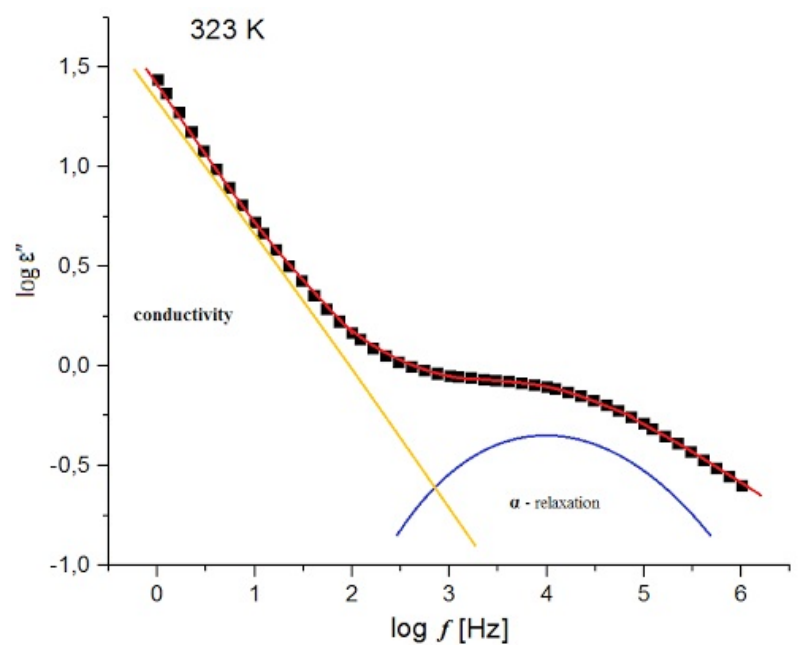

(b)

FIG. 4. Isothermal scans ( $\varepsilon^{\prime \prime}$ vs frequency) at different fixed temperatures for nanocomposite with 5 wt. $\%$ of nanosized $\mathrm{Cr}_{2} \mathrm{O}_{3}$. Characteristic temperatures for each process are chosen at which the process is visible in the measured frequency window. (a) $\gamma$ - and $\beta$ - relaxation, and (b) $\alpha$-relaxation. Symbols are the experimental data and full lines represent the total fit

In this equation, $\sigma_{0}$ is the dc conductivity and $\varepsilon_{0}$ is the permittivity of free space $(8.854 \mathrm{pF} / \mathrm{m})$. The fitting procedure is complicated very often because of the presence of incomplete peaks, in spite of the frequency window extending over more than 8 orders of magnitude. The quality of the fit is quite good and the characteristic relaxation time for each relaxation process can be extracted. The $\gamma$ - and $\beta$-relaxations are due to relatively shorter chain motions. The dependences of $-\log \tau_{\max }$ on the inverse temperature are linear for all nanocomposites and neat PA12 in the regions of $\gamma$ and $\beta$ processes (Fig. 5). As a result, the temperature dependence of these relaxations can be modeled by an Arrhenius type expression (4) [22]:

$$
\tau(T)_{\max }=\tau_{0} \exp \left(\frac{E_{a}}{R T}\right) .
$$

Here, $\tau_{0}=\tau_{\max }$ at $T \rightarrow \infty, E_{a}$ is the activation energy. Values of $\tau_{0}$ and $E_{a}$ are given in Table 1 .

The $\beta$-relaxation is phenomenologically linked to the mechanical properties of polymeric materials $[15,16]$. From Table 1, one can see that for the composites, both the $E_{a}$ and Young's modulus decrease. Assignment of molecular motions associated with the $\beta$-relaxation is complicated and a number of varying opinions exist in the literature $[18,19]$. However, these motions should be associated with the motion of amide groups together with

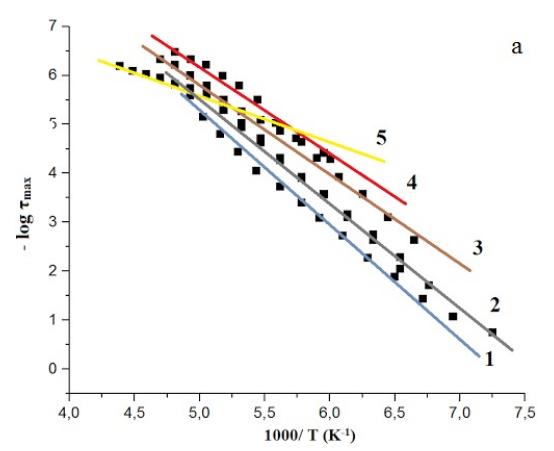

(a)

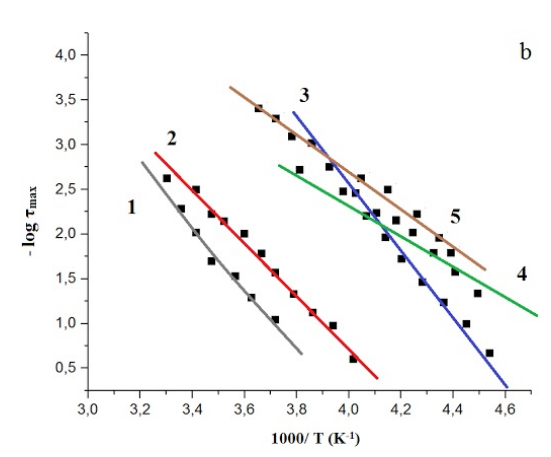

(b)

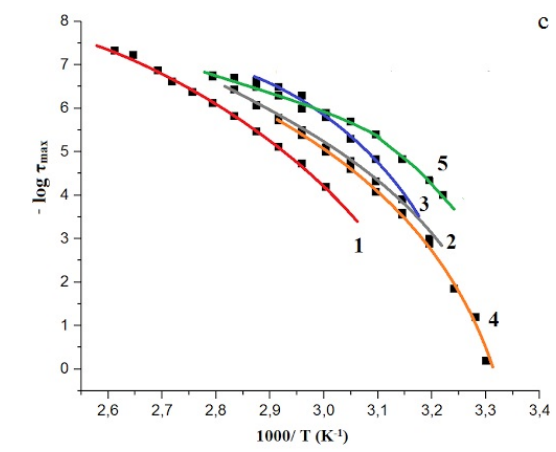

FIG. 5. Dependences of $-\log \tau_{\max }$ on the repciprocal of temperature for neat PA12 (1) and nanocomposites with 0.1 wt.\% (2); with 1 wt.\% (3); with 5 wt.\% (4); and with 10 wt.\% (5); (c) $\alpha$-relaxation; (b) $\beta$-relaxation; (a) $\gamma$-relaxation 
TABLE 1. $T_{g}$ and parameters of $\gamma, \beta$ and $\alpha$ (from VFT fit) relaxation processes of neat PA12 and nanocomposites, where $\tau_{0}$ is the relaxation time at infinite high temperature, $T_{0}$ is so-called Vogel temperature at which the relaxation time goes to infinity, and $D$ is the parameter related to the fragility of material

\begin{tabular}{|c|c|c|c|c|c|c|c|}
\hline $\begin{array}{c}\text { Samples, } \\
\text { wt. \% } \\
\mathrm{Cr}_{2} \mathrm{O}_{3} \\
\end{array}$ & & $E_{a}, \mathrm{~kJ} / \mathrm{mol}$ & $\tau_{0}, \mathrm{~s}$ & $D$ & $T_{0}, \mathrm{~K}$ & $T_{g}, \mathrm{~K}$ & $\begin{array}{l}\text { Young's } \\
\text { modulus, } \\
\text { GPa [8] }\end{array}$ \\
\hline \multirow[t]{3}{*}{0} & $\gamma$-mode & 83.7 & $3.9 \times 10^{-16}$ & & & & \\
\hline & $\beta$-mode & 59.2 & $4.4 \times 10^{-13}$ & & & & \\
\hline & $\alpha$-mode & 292.4 & $1.4 \times 10^{-7}$ & 3.32 & 280 & 332 & 0.97 \\
\hline \multirow[t]{3}{*}{0.1} & $\gamma$-mode & 62.7 & $3.9 \times 10^{-16}$ & & & & \\
\hline & $\beta$-mode & 48.3 & $3.9 \times 10^{-13}$ & & & & \\
\hline & $\alpha$-mode & 266.6 & $4.0 \times 10^{-10}$ & 4.25 & 253 & 329 & 0.66 \\
\hline \multirow[t]{3}{*}{1} & $\gamma$-mode & 80.0 & $3.1 \times 10^{-16}$ & & & & \\
\hline & $\beta$-mode & 60.25 & $3.6 \times 10^{-13}$ & & & & \\
\hline & $\alpha$-mode & 270.2 & $1.0 \times 10^{-10}$ & 2.95 & 267 & 318 & 0.49 \\
\hline \multirow[t]{3}{*}{5} & $\gamma$-mode & 71.4 & $4.3 \times 10^{-16}$ & & & & \\
\hline & $\beta$-mode & 59.4 & $2.3 \times 10^{-13}$ & & & & \\
\hline & $\alpha$-mode & 279.0 & $2.3 \times 10^{-10}$ & 3.10 & 265 & 315 & 0.47 \\
\hline \multirow[t]{3}{*}{10} & $\gamma$-mode & 67.0 & $3.8 \times 10^{-16}$ & & & & \\
\hline & $\beta$-mode & 52.6 & $4.9 \times 10^{-13}$ & & & & \\
\hline & $\alpha$-mode & 225.0 & $2.0 \times 10^{-9}$ & 2.47 & 256 & 303 & 0.36 \\
\hline
\end{tabular}

neighboring methylene groups. $\mathrm{Cr}_{2} \mathrm{O}_{3}$ is an amphoteric oxide which can undergo bonding with the amide group, therefore disrupting the H-bonding network between adjacent polymer chains. This leads to decrease in the $E_{a}$ of the $\beta$-relaxation and, hence, lowering in the Young's modulus. The decrease in the activation energy of the $\beta$-relaxation process indicates that the mobility of polymer matrix is a pre-condition for this mechanism to be effective.

The low temperature $\gamma$-relaxation involves the motion of short sequences of $\mathrm{CH}_{2}$ groups connected with an amide group which provides the dielectric activity. As a result, the dependence of $E_{a}$ of the $\gamma$-relaxation on the amount of nanofillers is quite.

The $\alpha$-relaxation is associated with onset of large-scale motions of the chain segments in the vicinity of $T_{g}$. The temperature dependence of the characteristic relaxation times can then be described using the Vogel-FulcherTammann equation [22]:

$$
\tau=\tau_{0} \exp \left(\frac{D T_{0}}{T-T_{0}}\right),
$$

where $\tau_{0}$ is the relaxation time at infinite high temperature, $T_{0}$ is so-called Vogel temperature at which the relaxation time goes to infinity, and $D$ is the parameter related to the fragility of material [23]. A smaller $D$ value implies a steeper temperature dependence for the relaxation time or a more "fragile" behavior. The $D$ data are given in Table 1. According to Plazek et al. [24,25] the activation energy of $\alpha$-relaxation process can be calculated using the following expression:

$$
\frac{E_{a}}{R}=\frac{D T_{0}}{\left(1-\frac{T_{0}}{T_{g}}\right)^{2}},
$$

where $E_{a}$ is the activation energy, $R$ is the gas constant and $T_{g}$ the glass transition temperature. The values of $D$ and $T_{0}$ parameters were extracted from the best fit to equation (3). The values of activation energies for $\alpha$-relaxation as well as the VFT parameters $T_{0}, D$ and $T_{g}$ are gathered in Table 1 . As can see, $T_{g}$ values of for 
the composites decrease in comparison to neat PA12, so the decrease in the moduli upon the introduction of the nanoparticles can be due to a plasticizing effect that the $\mathrm{Cr}_{2} \mathrm{O}_{3}$ nanoparticles could have exerted on the polymer matrix. However, $T_{g}$ of the composite with $0.1 \mathrm{wt} . \%$ of $\mathrm{Cr}_{2} \mathrm{O}_{3}$ is very similar (if not identical) to the $T_{g}$ of the pure polymer, but their Young's modulus is similar to moduli for other composites and is much smaller than that of pure PA12 [8], so the decrease in the moduli upon the introduction of the nanoparticles are not only due to a plasticizing effect. The softening of polymer matrix associated with local mobility ( $\beta$-relaxation) is probably more important. This effect is opposite to the typical result of nanofiller addition, and is manifested as the well-documented "antiplasticizing phenomenon" [26]. Because of their small size, nanofillers have a high surface-to-volume ratio and provide high-energy surfaces. A desired consequence of embedding nanosized fillers into a polymer matrix is the enhanced bonding between the matrix and additives. The composite theory predicts that improved bonding between polymer matrix and reinforcing phase leads to hindering of polymer chain motions [27]. Despite these predictions, however, BDS investigations of nanocomposites have provided mixed results [28]. We believe that this is caused by the presence of specific intermolecular interactions (e.g., H-bonding), within polymer matrix like PAs which can be influenced by nanoparticles. The nature of these interactions can depend upon both the polymer's and nanoparticles' chemical properties.

At temperatures above $\alpha$-relaxation, a MWS polarization [29] (Fig. 4) is observed. Moreover, at these temperatures, conductivity effects also play a role, and therefore, both the MWS process and conductivity phenomenon contribute to the high temperature dielectric response. To separate these processes is impossible, therefore they are not considered for further discussion.

\section{Conclusions}

In the present work, the molecular dynamics of nanocomposites based on PA12 filled with different loadings $(0.1-10$ wt.\%) of nanosized (average grain size of about $1-5 \mathrm{~nm}$ ) chromium (III) oxide was investigated by means of dielectric spectroscopy. For all polymer nanocomposites samples, two local relaxation modes, the $\gamma$ and $\beta$-relaxation, and a segmental $\alpha$-relaxation was observed. These relaxation modes were evaluated for further analysis. In addition, a high temperature response due to a MWS process combined with conductivity effects was noted.

The $E_{a}$ of the $\beta$-relaxation was shown to decrease for nanocomposites in comparison to neat PA12. The $\beta$ relaxation is phenomenologically linked to the mechanical properties of polymeric materials, hence, this observation can explain the Young's modulus decreases for these composites after nanofiller loading. The molecular reason for polymer matrix softening could be related to the disruption of amide H-bonds between neighboring polymer chains after complexation with nanosized chromium (III) oxide. The softening of the polymer matrix associated with local mobility ( $\beta$-relaxation) is supported by decreases in the glass transition temperatures related to the $\alpha$-relaxation for all nanocomposites. The Ea of the $\alpha$-relaxation also decreases, therefore, a plasticizing effect was observed upon the introduction of nanosized chromium (III) oxide into the polyamide matrix.

\section{References}

[1] Yan F., Li J., et al. Preparation of $\mathrm{Fe}_{3} \mathrm{O}_{4}$ /polystyrene composite particles from monolayer oleic acid modified $\mathrm{Fe}_{3} \mathrm{O}_{4}$ nanoparticles via miniemulsion polymerization.J. Nanopart. Res., 2009, 11, P. 289-296.

[2] Balakrishnan S.,, Bonder M.J., Hadjipanayis G.C. Particle size effect on phase and magnetic properties of polymer-coated magnetic nanoparticles. J. Magn. Magn. Mater, 2009, 321, P. 117-122.

[3] Giri S.K., Pradhan G.C., Das N. Thermal, electrical and tensile properties of synthesized magnetite/polyurethane nanocomposites using magnetite nanoparticles derived from waste iron ore tailing. J. Polym. Res., 2014, 21, 446.

[4] Kalia S., Kango S., et al. Magnetic polymer nanocomposites for environmental and b iomedical applications. Colloid. Polym. Sci., 2014, 292, P. 2025-2052.

[5] Vollenberg P.H.T., Heikens D. Particle size dependence of the Young's modulus of filled polymers: 1. Preliminary experiments. Polymer, 1989, 30, P. 1656-1662.

[6] Gersappe D. Molecular Mechanisms of Failure in Polymer Nanocomposites. Phys. Rev. Lett., 2002, 89, 058301(1-4).

[7] Dos Santos L.R., Ligabue R., et al. New magnetic nanocomposites: Polyurethane/Fe ${ }_{3} \mathrm{O}_{4}$-synthetic talc. Eur. Polym. J., 2015 , 69, P. 38-49.

[8] Shapoval E.S., Zuev V.V. Novel polyamide 12/ chromium (III) oxide nanoparticles composites: melting behaviour and complex isothermal crystallization kinetics. Polymer Composites, 2015, 36 (6), P. 999-1005.

[9] Jordan J., Jacob K.I., et al. Experimental trends in polymer nanocomposites?a review. Mater. Sci. Eng. A, 2005, 393, P. 1-11.

[10] Zhu L., Narh K.A. Numerical simulation of the tensile modulus of nanoclay-filled polymer Composites. J. Polym. Sci. Part B. Polym. Phys., 2004, 42, P. 2391-2406.

[11] Ciprari D., Jakob K., Tannenbaum R. Characterization of polymer nanocomposites interphase and its impact on the mechanical properties. Macromolecules, 2006, 39, P. 6565-6573.

[12] Kango S., Kalia S., et al. Surface modification of inorganic nanoparticles for development of organic?inorganic nanocomposites?A review. Prog. Polym. Sci., 2013, 38, P. 1232-1261. 
[13] Allegra G., Raos G., Vacatello M. Theories and simulations of polymer based nanocomposites: from chain statistics to reinforcement. Prog. Polym. Sci., 2008, 33, P. 683-731.

[14] Adachi K., Kotaka T. Dielectric normal mode relaxation. Progr. Polym. Sci., 1993, 18, P. 585-622.

[15] Boyer R.F. Dependence of mechanical properties on molecular motion in polymers. Polym. Eng. Sci., 1968, 8, P. 161-185.

[16] Liu H., Brinson L.C. Reinforcing efficiency of nanoparticles: a simple comparison for nanocomposites. Compos. Sci. Tech., 2008, 68, P. $1502-1512$.

[17] Rathmanathan K., Cavaille J.Y., Johari J.P. The dielectric properties of dry and water- Saturated nylon 12. J. Polym. Sci. Part B. Polym. Phys., 1992, 30, P. 341-348.

[18] Laredo E., Hernandez M.C. Moisture effect on the low- and high-temperature dielectric relaxations in nylon-6. J. Polym. Sci. Part B. Polym. Phys., 1997, 35, P. 2879-2888.

[19] Starkweather Jr.H.W., Avakian P. Conductivity and the electric modulus in polymers. J. Polym. Sci. Part B. Polym. Phys., 1992, 30, P. 637-641.

[20] Havriliak S., Negami S., A complex plane analysis of $\alpha$-dispersions in some polymer systems. J. Polym. Sci. Part C, 1966,14 , P. 99-117.

[21] Havriliak S., Negami S., A complex plane representation of dielectric and mechanical relaxation processes in some polymers. Polymer, 1967, 8, P. 161-210.

[22] Kremer F., Schonhals A. Broadband dielectric spectroscopy. Springer, New York, 2003.

[23] Angell C.A., Ngai K.L., et al. Relaxation in glassforming liquids and amorphous solids. J. Appl. Phys., $2000, \mathbf{8 8 ,} 3113$.

[24] Plazek D.J., Magil J.H. Physical properties of aromatic hydrocarbons I. J. Chem. Phys., 1998, 35, P. 3038-3050.

[25] Bureau E., Cabot C., Marais S., Saiter J.M. Study of the ?-relaxation of PVC, EVA and 50/50 EVA70/PVC blend. Eur. Polym. J., 2005, 41, P. 1152-1158.

[26] Treacy M.M.J., Ebesen T.W., Gibson J.M. Nanoparticles as reinforced agents. Nature (London), 1996, 381, P. 678-680.

[27] Schaefer D.W., Justice R.S. How nano are nanocomposites. Macromolecules, 2007, 40, P. 8501-8517.

[28] Sinitsin A.N., Zuev V.V. Dielectric relaxation of fulleroid materials filled PA6 composites and the study of its mechanical performance. Nanosystems: physics, chemistry, mathematics, 2015, 6, P. 570-?582.

[29] Baird M.E., Goldworthy G., Creasey C.J. Low frequency dielectric behavior of polyamides. Polymer, 1971, 12, P. 159-175. 